\title{
Posición de la Sociedad Argentina de Hipertensión Arterial sobre la ingesta de sodio como componente de las aguas naturales y su implicancia sobre la presión arterial
}

Position of the Argentinian Society of Arterial Hypertension about sodium intake as a component of commercialized mineral waters and implications on arterial blood pressure

Trabajo presentado en el XXI Congreso de Hipertensión Arterial 2014

Felipe Inserra ${ }^{1 a}$, Martín Salazar ${ }^{2 b}$, Alfredo Wassermann ${ }^{3 b}$, Gustavo Lavenia ${ }^{4 b}$, Roberto Miatello ${ }^{5 b}$, Nicolás Renna ${ }^{6 b}$, Jorge Janson ${ }^{7 b}$, Fernando Filippinisb

\section{RESUMEN}

Las aguas naturales son una fuente de aporte de sales. Los aportes de estos minerales con las aguas varían según el origen y composición de estas.

El sodio aportado por las aguas naturales se encuentra mayoritariamente formando sales con bicarbonato y sulfato y no con cloruro (responsable del aumento de la presión arterial). Asimismo, estas aguas aportan otros minerales, de los cuales calcio, magnesio y potasio son los más importantes. Sobre estos últimos. la información referida a la evaluación de sus efectos sobre la presión arterial y la enfermedad cardiovascular parece confirmar que sus efectos no son considerados perjudiciales a las concentraciones y cantidades que suelen ser ingeridos, sino por el contrario podrían generar beneficios sobre la salud y el riesgo cardiovascular.

En cuanto a la magnitud relativa del sodio en estas aguas, es porcentualmente baja cuando se lo compara con lo que habitualmente se ingiere con el resto de los alimentos. Precisamente, la cantidad máxima de sodio aportada por estas aguas es menos del 10\% del consumo máximo recomendado por la Organización Mundial de la Salud. Esto implica entonces que el aporte de sodio proveniente del agua es poco relevante dentro de una alimentación variada, teniendo en cuenta además que la mayor parte del mismo no es cloruro de sodio, sino otras sales como bicarbonato y sulfato de sodio, con un efecto al menos neutro sobre la presión arterial.

\begin{abstract}
Natural waters are a source of salts input. The content of these waterborne minerals will vary depending on water origin and composition.

In natural water sodium is mainly present as bicarbonate salts rather than sodium chloride (the compound leading to hypertension). Likewise, there are other minerals in water, of which the most important are calcium, magnesium and potassium The latest information on these minerals seems to confirm that their effects on blood pressure and cardiovascular disease are not harmful at the amounts and concentrations at which they tend to be ingested: on the contrary, they may even be beneficial for cardiovascular function and general health.

Relative sodium content in natural waters is low in percentage terms if compared with what consumers tend to ingest with food. In fact, the maximum amount of sodium content in water accounts for less than $10 \%$ of the maximum consumption recommended by the World Health Organization. This means that sodium intake from water is irrelevant in a balanced diet, as waterborne sodium is present not as sodium chloride, but as other salts such as sodium bicarbonate and sulfate, which produce different effect on blood pressure.
\end{abstract}

Keywords: hypertension, sodium, natural water.

Palabras clave: hipertensión, sodio, agua natural.

b. Coordinación de Contenidos

1. Presidente de la Sociedad Argentina de Hipertensión Arterial. Co-Director de la Maestría de Mecánica Vascular e Hipertensión Arterial de la Universidad Austral.

2. Jefe de Servicio de Clínica Médica, Hospital Universitario San Martín de La Plata. Docente de la Cátedra“D” de Medicina Interna, Universidad Nacional de La Plata.

3. Jefe de Nefrología y Presidente del Comité de Hipertensión, Hospital Prof. Dr. B. A. Houssay. Director Médico de FEPREVA.

4. Maestría de Hipertensión Arterial y Mecánica Vascular de la Universidad Austral. Ex Presidente Asociación de Hipertensión Arterial de Rosario.

5. Profesor Adjunto de Fisiología Patológica, Facultad de Ciencias Médicas, Universidad Nacional de Cuyo Investigador Independiente CONICET.

6. Jefe de trabajos Prácticos Departamento de Patología. Facultad de Ciencias Médicas. Universidad Nacional de Cuyo. Investigador asistente IMBECU-CONICET.

7. Servicio de Clínica Médica y Sección Hipertensión Arterial. Hospital Italiano. Director del Curso: Habilidades Narrativas para Médicos. Instituto Universitario Hospital Italiano

8. Vicepresidente Primero de la Sociedad Argentina de Hipertensión Arterial. Profesor Adjunto de Clínica Médica y Terapéutica de la Univ. Nacional de Rosario. Profesor Titular de Fisiopatología Adultos I y II. Carrera de Nutrición Universidad Abierta Interamericana. 


\section{INTRODUCCIÓN}

La adecuada y fina regulación del equilibrio hidroelectrolítico de los seres vivos es lo que ha posibilitado que ellos se adapten a la vida fuera del agua de los ríos y mares de nuestro planeta, al evitar la deshidratación y mantener la adecuada composición hidrosalina de los organismos. Este hecho, que evolutivamente en las especies implica millones de años de cambios, adaptaciones y supervivencia de los más aptos, se ha producido gracias al desarrollo de vías y mecanismos mediadores que interactúan entre los sistemas o aparatos, y en los distintos órganos, tejidos, células e incluso, en las organelas subcelulares. Estos mecanismos resultantes de la evolución son muy eficientes, redundantes y complementarios, y aquellos que regulan la reabsorción para la conservación del agua y los electrolitos constituyen el mejor ejemplo de esta adaptación evolutiva que posibilitó la vida tal como hoy la conocemos.

El agua es el componente esencial de los seres vivos, ya que entre el 60 y $70 \%$ del peso corporal (unos 45 a 50 litros) está constituido por agua. Una deshidratación que alcance al $10 \%$ del líquido corporal suele producir la muerte, lo mismo que cambios porcentuales pequeños de la composición electrolítica. Las comidas aportan entre 500 y $900 \mathrm{ml}$ de agua por día, mientras que las bebidas proveen entre 1000 y $1500 \mathrm{ml}$ más al día, dependiendo esto de la temperatura, actividad física y agua aportada por los alimentos. Se generan también unos $350 \mathrm{ml}$ por día de agua, proporcionada por el metabolismo de los alimentos conocida como agua metabólica. Los otros elementos clave que aportan al fino equilibrio mencionado inicialmente son los minerales, que en general ingresan acompañando a los alimentos y al agua ingerida, variando la cantidad y variedad del tipo y composición de los mismos.

El sodio representa el catión más abundante en la composición de los líquidos extracelulares y se encuentra asociado formando dos sales esenciales: cloruro y bicarbonato de sodio. Estos abundantes compuestos tienen la responsabilidad primaria de mantener dos funciones esenciales para los seres vivos, la osmolaridad de los líquidos y su equilibrio ácido-base y el de las células. Como veremos en el desarrollo, la alta ingesta crónica de cloruro de sodio por parte de los mamíferos, incluidos los seres humanos, se acompaña de aumento de la presión arterial y también de mayor porcentaje de individuos desarrollan hipertensión arterial. Esto es un hecho epidemiológico muy relevante, teniendo en cuenta que el elevado consumo actual de cloruro de sodio por la enorme mayoría de los seres humanos, que es varias veces superior a las necesidades vitales, está asociada con una carga mayor de hipertensión arterial y de enfermedades cardiovasculares. Sin embargo, otras sales de sodio como el bicarbonato parecen funcionar de diferente manera, existiendo algunas evidencias de que la responsabilidad principal parece depender del cloruro que acompaña al sodio o la sal en sí misma, y no el sodio en forma aislada o formando parte de otras sales como el bicarbonato de sodio. Las aguas naturales son una fuente de aporte de sales, especialmente de bicarbonato de sodio, como también sales de calcio y magnesio, entre otras. Los aportes de estos minerales con las aguas varían de acuerdo con el origen y composición de estas. Sin embargo, la magnitud relativa de algunos de los minerales aportados, especialmente la del sodio, es porcentualmente baja cuando se la compara con lo que habitualmente se ingiere con el resto de los alimentos.

Adicionalmente, existen dos hechos importantes que vale la pena comentar:

1. El sodio aportado por las aguas naturales se encuentra mayoritariamente formando sales con bicarbonato y no con cloruro; también estas aguas aportan otros minerales, de los cuales calcio, magnesio y potasio son los más importantes. Sobre estos últimos, la información referida a la evaluación de sus efectos sobre la presión arterial y la enfermedad cardiovascular parece confirmar que sus efectos no son considerados perjudiciales a las concentraciones y cantidades que suelen ser ingeridos, sino por el contrario podrían generar beneficios sobre la salud y el riesgo cardiovascular.

2. Por otra parte, la ingestión de agua es reconocida como la mejor manera de brindar el aporte necesario para la adecuada hidratación y balance hidroelectrolítico de nuestro organismo. Asimismo, el hábito de tomar agua hace que se modere o evite la ingestión de otras bebidas que, en cambio, son reconocidos factores de riesgo, tanto para el desarrollo y mantenimiento de hipertensión arterial como para la enfermedad cardiovascular. Nos referimos al exceso de bebidas alcohólicas, las bebidas azucaradas o las llamadas "energizantes".

Estos conceptos preliminares motivaron a la Sociedad Argentina de Hipertensión Arterial para revisar la información científica existente, así como generar y dar a conocer esta Toma de Posición sobre Ingesta de sodio como componente de las aguas naturales a los profesionales, pacientes y población en general.

Un hecho que nos parece muy relevante del documento es que las distintas consideraciones fueron vertidas por los expertos con mucha cautela, dado que la mayor parte de la abundante información revisada proviene de estudios observacionales, o de cohortes o metaanálisis, y que no existen estudios de intervención controlados que otorguen a la información la fortaleza necesaria para generar recomendaciones o afirmaciones sobre conductas prescriptivas. Si rotulásemos los conceptos según se hace habitualmente en las guías, muchos de ellos serían "opinión de expertos". Sin embargo, van a notar que, luego de una adecuada revisión de la información disponible, en algunos 
casos la misma da soporte a conceptos arraigados en el conocimiento médico, mientras que, en otros casos, también sirve, pero para desmitificar algunas creencias populares incluidas las de algunos profesionales. Es posible, y también es nuestro deseo, que este documento contribuya a estimular la necesidad de mejorar del conocimiento científico acerca de la importancia que tiene la composición de las aguas de consumo sobre la salud humana.

\section{PREGUNTAS GENERADAS EN LA TOMA DE POSICIÓN}

1. ¿Cuál es la influencia de la ingesta de sodio sobre la presión arterial?

2. Aguas con diferentes contenidos de sodio. ¿Cuál es la relevancia de la cantidad diferencial del sodio aportado diariamente por su ingesta?

3. ¿Cuál es la importancia sobre la presión arterial de los distintos aniones que acompañan al sodio?

4. ¿Cómo influyen los otros minerales presentes en las aguas naturales sobre la presión arterial?

5. ¿Cuál es la implicancia del hábito de tomar agua y no otras bebidas sobre la presión arterial?

6. ¿El sabor del agua según el contenido de minerales influye sobre la adherencia a su consumo habitual?

\section{1. ¿Cuál es la influencia de la ingestión de sodio sobre la presión arterial?}

- La relación entre ingesta de cloruro de sodio, o sal de mesa, y elevación de la presión arterial (PA) es sostenida por evidencia de diversas fuentes.

- Estudios epidemiológicos muestran una asociación entre consumo de sodio como cloruro, PA media de la comunidad y prevalencia de hipertensión arterial (HTA $)^{1,2}$. La HTA es muy infrecuente en comunidades primitivas que consumen menos de $50 \mathrm{mEq} / \mathrm{día}$, aunque otros factores como el bajo consumo de alcohol, la ingesta elevada de potasio y la ausencia de obesidad podrían interactuar. Es posible que el desarrollo de HTA esencial requiera un determinado umbral de consumo de sal ${ }^{3}$.

- Estudios experimentales en mamíferos describen una relación positiva entre el consumo de cloruro de sodio y la elevación de la PA; el efecto del cloruro de sodio tiene una fase aguda y una crónica progresiva, y se ha señalado que una vez pasado cierto tiempo, disminuir el consumo reduce, pero no regresa los valores de PA a los iniciales, probablemente por haber puesto en marcha otros mecanismos que tienden a aumentar la PA, como el estrés oxidativo y los subsecuentes procesos inflamatorios ${ }^{4-8}$.

- Los lactantes con baja ingesta de sal tienen PA más bajas y las presiones durante la in- fancia podrían determinar en parte los valores de PA durante la vida adulta9,10.

- El cloruro de sodio parece estar implicado en el incremento de la PA con la edad, aumento que puede atenuarse con una disminución de la ingesta de $\mathrm{sal}^{11}$.

- Ensayos clínicos y metaanálisis demostraron que disminuir la ingesta de cloruro de sodio reduce la $\mathrm{PA}^{12-15}$. Un reciente metaanálisis ha estimado que disminuir la ingesta diaria del sodio en $75 \mathrm{mEq}$ por más de 4 semanas reduce la PA (sistólica/diastólica) $5 / 3 \mathrm{mmHg}$ en hipertensos y $2 / 1 \mathrm{mmHg}$ en normotensos. La disminución de la PA con la reducción en la ingesta de cloruro de sodio muestra una relación dosis-respuesta. Además, reducir la ingesta de sal mejora la eficacia de los antihipertensivos, particularmente diuréticos, IECA y ARA $2^{16-20}$.

- Existen también evidencia de que si se modifica la composición de la sal ingerida, reduciendo el contenido de cloruro de sodio y remplazándolo parcialmente por cloruro de potasio y sulfato de magnesio se consigue disminuir la presión arterial y reducir los eventos cardiovasculares ${ }^{21}$.

- Evidencias experimentales podrían acercar una explicación fisiopatológica coherente a la relación entre mayor ingesta de cloruro de sodio e HTA: a) en los pacientes hipertensos existe un aumento del sodio intracelular en paredes vasculares, células sanguíneas y cardiomio$\operatorname{citos}^{22-24}$; b) el aumento del sodio intracelular podría actuar aumentando el calcio intracelular y consecuentemente estimular vías de señalización intracelular dependientes de calcio y también por la activación de la quinasa-1 sal sensible (SIK1) disparando mecanismos de vasoconstricción, de hipertrofia y de proliferación celular $^{25}$; c) El aumento de sodio intracelular se debería a la hiperactividad del intercambiador $\mathrm{Na}^{+} / \mathrm{H}^{+}(\mathrm{NHE}-1)^{26}$, a la presencia de una sustancia endógena similar a la ouabaína ${ }^{27}$ o bien del trasporte $\mathrm{Na}^{+}, \mathrm{HCO}^{-}$dependiente del cotransportador $\mathrm{NBCn}^{28}$.

- Recientemente se han encontrado elementos que sugieren que el cotransportador NBCn1 de $\mathrm{Na}^{+}, \mathrm{HCO}^{-}$mediante el control del $\mathrm{pH}$ intracelular y consecuentemente la actividad enzimática, especialmente de los canales iónicos, juega un rol crítico en el mantenimientodel tono vascular, mediante la regulación de la función de las células musculares lisas vasculares y de las células endoteliales ${ }^{29}$. Se podría especular que fenómenos vinculados a estos mecanismos son una posible explicación de por qué el sodio como bicarbonato tiene un efecto diferente al sodio como cloruro sobre el comportamiento vascular. 


\begin{tabular}{|c|c|c|c|c|c|c|c|c|c|}
\hline \multirow{2}{*}{$\begin{array}{l}\text { Marcas } \\
\text { nacionales }\end{array}$} & Sodio & Potasio & Calcio & Magnesio & Bicarbonato & Sulfatos & Cloruros & Fluoruros & Sílice \\
\hline & \multicolumn{9}{|c|}{ Todos en mg/l } \\
\hline Villa del Sur * & 164 & 10 & 19 & 12 & 450 & 29,8 & 37,5 & 0,7 & \\
\hline Villavicencio * & 110 & 4,8 & 43,7 & 45,3 & 384 & 148 & 27,6 & 1,18 & 22,3 \\
\hline Eco de los Andes* & 10 & 4 & 30 & 3 & 79,3 & 0 & 44,2 & 1,1 & \\
\hline Pureza Vital * & 79,2 & 0 & 51,5 & 5,2 & 225,3 & 0 & 0 & 0,8 & \\
\hline Glaciar * & 10 & 45 & 40 & 4 & 79 & 15 & 70 & 0,5 & \\
\hline Bon aqua * & 35 & 0 & 39 & 4,9 & 109 & 47 & 36 & & \\
\hline Dasani * & 5 & 1,5 & 0 & 3 & 0 & 15 & 10 & 0 & \\
\hline Sierra de los Padres * & 187 & 8 & 41 & 15 & & & & 0,4 & \\
\hline Día * & 18,6 & 0 & 88,7 & 23,4 & 297,2 & 43,9 & 35,8 & & 7,1 \\
\hline \multicolumn{10}{|c|}{ * Datos tomados de etiquetas de producto mercado durante el mes de julio de 2013} \\
\hline \multicolumn{10}{|l|}{$\begin{array}{l}\text { Marcas } \\
\text { internacionales }\end{array}$} \\
\hline Evian & 5 & 1 & 78 & 24 & 357 & 10 & 4,5 & & 13,5 \\
\hline San Peregrino & 33 & 2,5 & 170 & 52 & 200 & 450 & 52 & 0,56 & \\
\hline Perrier & 11,8 & 0 & 155 & 6,8 & 445 & 46,1 & & & \\
\hline
\end{tabular}

- Así el cloruro de sodio o sal común, parece cumplir con los criterios de Bradford Hill de causalidad de aumento de la PA y la mayoría de las guías actuales recomiendan una reducción moderada de la ingesta de cloruro de sodio para la población en general y para los pacientes hipertensos en especial ${ }^{30-32}$. Aunque no hay unanimidad en el nivel de ingesta recomendado, estaría en el rango de 3-6 gr de sal por día. La OMS recomienda que sea inferior a 5,5 g/día. Además de su influencia sobre la presión arterial, el aumento de la ingesta de sal con la dieta ha sido relacionado con la masa ventricular izquierda ${ }^{33}$ y con la mortalidad cardiovascular ${ }^{34}$.

\section{Aguas con diferentes contenidos de sodio. ¿Cuál es la relevancia de la cantidad diferen- cial del sodio aportado diariamente por su ingesta?}

- El primer punto que queremos resaltar es que los contenidos de minerales de las aguas reportados en las etiquetas de los productos es en $\mathbf{m g}$ por litro, y no en $\mathbf{m g}$ cada $\mathbf{2 0 0 ~} \mathbf{m l}$ como se expresan las concentraciones en el resto de bebidas y alimentos envasados, según el código alimentario nacional. Esto puede generar algunas confusiones inclusive en algunos profesionales.

- Los principales elementos que pueden contener las aguas naturales son calcio, magnesio, sodio y potasio. Con concentraciones máximas que pueden alcanzar 730, 450, 1900, y $270 \mathrm{mg} / \mathrm{L}$, respectivamente, sin embargo las concentraciones habituales en las aguas suelen ser mucho más bajas ${ }^{35}$.

- El aporte del sodio por las aguas naturales suele influir poco en la cantidad total ingerida de sodio, y sólo ante una ingesta abundante de aguas con alto contenido del ion podrían tener alguna relevancia. El consumo diario de sal es de aproximadamente 10-12 g por día de promedio en los adultos. En un estudio hecho en nuestro país por el Ministerio de Salud de la $\mathrm{Na}$ ción, evaluando la excreción urinaria de sodio se encontró que el promedio de consumo de sal en es de 11,2 gramos por día (siendo de $12,7 \mathrm{~g}$ en hombres y de $9,8 \mathrm{~g}$ en mujeres) ${ }^{36}$. El $75-80 \%$ del aportede cloruro de sodio proviene de la sal contenida en los alimentos procesados.

- Los beneficios derivados de la ingestión de agua mineral natural tienen un doble origen, uno es el aporte de agua y su consiguiente papel sobre la hidratación, y el segundo es el aporte de minerales ${ }^{37}$.

- De la cantidad de sodio y otros minerales, con sus respectivas sales, depende el grado de mineralización de las aguas. Esto comprende el contenido global en minerales del agua, también llamado residuo $\operatorname{seco}^{38}$.

- La clasificación de contenidos minerales según nuestro código alimentario nacional en el art. 986, y que es la que rige para el etiquetado, es:

1. de acuerdo al grado de mineralización determinado por el residuo seco soluble:

a. oligomineralizada: residuo entre $50 \mathrm{y}$ $100 \mathrm{mg} / \mathrm{l}$.

b. de mineralización débil: residuo entre 101 y 500 .

c. de mineralización media: residuo entre 501 y 1500 .

d. de mineralización fuerte: residuo entre 1501 y 2000.

2. el mismo código, en relación al contenido de sodio, considera como

a. bajas en sodio cuando la concentración es de hasta $20 \mathrm{mg} / \mathrm{L}$.

b. sódicas las que contienen una concentración mayor de $200 \mathrm{mg} / \mathrm{L}$.

c. podríamos considerar como de contenido intermedio cuando el contenido de sodio es entre 20 y $200 \mathrm{mg} / \mathrm{L}^{39}$. 
A manera de ejercicio, considerando un agua sódica que brinda un aporte de $200 \mathrm{mg} / \mathrm{l}$ del ion sodio, y suponiendo una ingesta de $1500 \mathrm{ml}$ en el día, la cantidad de sodio aportado alcanza a $300 \mathrm{mg}$ diarios. Teniendo en cuenta que, en nuestro medio, el consumo diario de sodio oscila entre los 4,5 a $5 \mathrm{~g}$ (equivalente a 11 a $12 \mathrm{~g}$ de cloruro de sodio), el aporte posible por este tipo de aguas constituye menos del $7 \%$ del total del consumo diario habitual de sodio, y alrededor del $10 \%$ de la cantidad de sodio recomendado por la OMS. Es por esta razón, que la OMS en su reciente guía sobre calidad de agua afirma que no está demostrada una asociación entre el contenido de sodio de las aguas y la ocurrencia de hipertensión, y en tal sentido no da ningún valor de referencia de la cantidad de sodio relacionado a la salud, sólo menciona que puede afectar el sabor del agua potable en niveles superiores a $200 \mathrm{mg} / \mathrm{litro}^{40}$.

Es decir, el aporte de sodio proveniente del agua es poco relevante dentro de una alimentación variada, y además la mayor parte del mismo no es cloruro de sodio.

\section{3. ¿Cuál es la importancia sobre la presión arte- rial de los distintos aniones que acompañan al sodio?}

- Los principales aniones que acompañan al sodio en las aguas de consumo son el bicarbonato, sulfato y cloruro ${ }^{35}$.

- Las aguas naturales que se originan en manantiales, carbonatadas o no, suelen contener cantidades variables de sodio, acompañadas esencialmente por los aniones bicarbonatoy sulfato. Existe bastante evidencia sobre que el efecto en la presión arterial que tienen las sales de sodio parece depender esencialmente del anión acompañante. Según diferentes estudios, los efectos sobre la PA por cantidades comparables de sodio ingerido resultó diferente dependiendo de que los aniones acompañantes fuesen cloro o bicarbonato. Asimismo, existe información experimental sobre el aporte de cloruros libres de sodio constituyendo otras sales, que muestra que el aporte de cloruro produce aumento de la PA y también del número de eventos vasculares en los animales ${ }^{41}$. Esta información incrementa la importancia del anión cloruro, independientemente del sodio, sobre su participación al desarrollo y mantenimiento de la hipertensión arterial ${ }^{142}$.

- El efecto del bicarbonato de sodio sobre la PA es diferente del provisto por cantidades equivalentes de cloruro sódico ${ }^{43,44}$. Es así que el aporte de bicarbonato de sodio, en comparación con cantidades iguales de sodio aportadas como cloruro produce descenso de la presión arterial y disminución del riesgo cardiovascular ${ }^{45,46}$. Inclusive existe información preliminar que refie- re que el bicarbonato de sodio mejora el perfil lipídico de los pacientes ${ }^{47,48}$.

- En un estudio de 7 semanas, en pacientes entre 24 y 53 años de ambos géneros, que recibieron aguas minerales con sodio unido al anión bicarbonato, pudieron prevenir el incremento de la presión arterial comparativamente con las que recibían igual cantidad de sodio pero como cloruro; la ingesta de $500 \mathrm{ml}$ de agua mineral rica en bicarbonato de sodio no generó modificaciones en la $\mathrm{PA}^{45}$. Este efecto no fue verificado en hipertensos "sal sensibles". Estos resultados podrían estar vinculados, por lo menos en parte, a que el aumento de la PA y la sal-sensibilidad parecen depender del anión cloruro ${ }^{41}$.

- Además la posible modificación del pH intracelular, como resultado del consumo de bicarbonato en lugar de cloruro, puede ser unos de los mecanismos responsables del efecto diferencial de dichos aniones, mediado por una atenuación del tono vascular y consecuentemente de la presión arterial ${ }^{29}$.

- También se ha encontrado que las sales de bicarbonato de sodio tendrían un rol protector sobre la filtración glomerular y el tono vasocontrictor de la vasculatura renal, pudiendo atenuar o evitar la aparición y mantenimiento de la $\mathrm{HTA}^{49-50}$.

\section{4. ¿Cómo influyen los otros minerales presen- tes en las aguas naturales sobre la presión arterial?}

- La concentración de minerales en las aguas, incluyendo las de sales de sodio, calcio magnesio y potasio,varía según la fuente u origen de las aguas para cuando se trate de aguas naturales, o bien de la fórmula comercial para las aguas mineralizadas embotelladas o de red.

- Existe gran variabilidad en la composición mineral de las diferentes aguas minerales envasadas en el mundo ${ }^{51}$. El agua de red tiene composiciones diversas de acuerdo con su origen y su procesamiento.

- Las diversas fuentes que tiene la totalidad del agua que bebemos a diario los seres humanos, junto a la importante variación en el tipo de alimentos ingeridos, dificultan la posibilidad de establecer un vínculo certero entre determinado producto, sus componentes minerales y sus eventuales efectos sobre la salud.

- Si bien existen estudios que relacionan la ingesta de algunos minerales con la hipertensión arterial, el riesgo de eventos y la mortalidad cardiovascular, la certeza y la calidad de los datos sobre la cantidad de minerales ingeridos en forma diaria en muchos de ellos no permite tener 
evidencias definitivas ${ }^{52-54}$. Si bien el aporte de ciertos minerales no sódicos provenientes de las aguas podrían contribuir a efectos beneficiosos sobre la presión arterial y la morbimortalidad cardiovascular, la información no es aún lo suficientemente uniforme ${ }^{55}$.

- Los minerales no sódicos, que con más frecuencia han sido vinculados a modificaciones de la presión arterial y a variaciones en el riesgo y la incidencia de eventos cardiovasculares son esencialmente calcio, magnesio y potasio ${ }^{56-58}$. Son varios los estudios que evaluaron los potenciales beneficios de ingerir aguas naturales que contienen estos minerales sobre la PA y el riesgo cardiovascular ${ }^{59-60}$.

- Magnesio: algunos trabajos muestran beneficios consistentes sobre la PA incorporar cationes, principalmente magnesio, particularmente cuando su consumo diario es insuficiente ${ }^{61}$. La deficiencia de magnesio ha sido implicada en la patogénesis de la hipertensión y varios estudios epidemiológicos y experimentales muestran que la baja ingesta de magnesio puede incrementar el riesgo de enfermedades cardiovasculares y accidente cerebro vascular ${ }^{62,63}$. Existen también estudios que muestran que tanto ingestas deficitarias como niveles plasmáticos bajos de magnesio aumentan la prevalencia de muerte súbita ${ }^{64}$. Asimismo, los bajos niveles de magnesio se asociaron con la presencia de HTA y también con alteraciones en la estructura vascular ${ }^{65,66}$.

Asimismo, se ha descripto que los productos y las aguas que contienen mayor cantidad de magnesio producen una reducción de la enfermedad coronaria, particularmente una mayor ingesta de magnesio reduce los eventos coronarios fatales ${ }^{51,67}$. Existe una correlación negativa entre niveles de magnesio plasmático y la presión arterial ${ }^{68}$. Los estudios clínicos que evaluaron aportes complementarios de magnesio han tenido resultados en su mayoría favorables, aunque sus resultados son menos uniformes y consistentes $^{69.70}$. Si parece convincente que el suministro de agua conteniendo magnesio redujo la PA en aquellas personas cuyos niveles urinarios, como expresión del consumo de magnesio, eran bajos. Las poblaciones que habitualmente ingieren aguas duras, con elevado contenido en calcio y magnesio, tienen menor mortalidad cuando se comparan con otras poblaciones que ingieren aguas con bajo contenido mineral. La relación entre la presencia de magnesio en el agua de consumo y el riesgo de ECV ha sido revisada en un metaanálisis ${ }^{70}$, que concluyó que concentraciones de magnesio en el agua, entre 8,3 y $19,4 \mathrm{mg} / \mathrm{L}$ se asocian con menor morta- lidad cardiovascular, aunque existealgún trabajoque no ha podido confirmarlo ${ }^{71}$. En el mismo sentido, el suplemento oral de magnesio mostró un efecto dosis-dependiente sobre la reducción de la PA. La disminución fue de 4,3 $\mathrm{mmHg}$ en la PA sistólica y $2,3 \mathrm{~mm} \mathrm{Hg}$ en la PA diastólica por cada $10 \mathrm{mmol}(240 \mathrm{mg}) /$ día de incremento en la ingesta de magnesio ${ }^{72}$. En un metaanálisis reciente se encontró que existe una correlación negativa entre la ingesta de magnesio y la incidencia de accidente cerebrovascular isquémico $^{73}$.Los mecanismos propuestos por los cuales el magnesio generaría el beneficio son:

a. activación de la $\mathrm{Na}^{+} / \mathrm{K}^{+}$-ATPasa;

b. bloqueo de los canales de calcio;

c. regulación del potasio, sodio y calcio intracelular;

d. efecto depresor del tono actuando sobre el músculo liso vascular;

e. reducción del estrés oxidativo y de la agregación plaquetaria ${ }^{61}$

Sin embargo, esta información disponible no es suficiente para generar una recomendación sobre posibles beneficios de las sales de magnesio aportadas por las aguas. Para confirmar estos datos hacen falta estudios de intervención, adecuadamente diseñados.

- Calcio: el efecto favorable para las aguas con mayor contenido de calcio ha sido también reportado en varios trabajos, aunque de manera menos constante ${ }^{60}$. Algunos trabajos señalan que la suplementación aislada de calcio no conferiría beneficios cardiovasculares ${ }^{74,75}$ ni modificaría las cifras de $\mathrm{PA}^{76}$. Sin embargo otros autores refieren que se reducen las cifras de PA, y que también disminuyen la incidencia de $\mathrm{ACV}^{77,78}$. En el mismo sentido, junto a la ingesta de vitamina $D$, podrían reducir la PA y también la frecuencia cardíaca en pacientes año$\operatorname{sos}^{79}$. Podríamos concluir, en cuanto a las sales de calcio, que para confirmar un potencial efecto protector son necesarias más investigaciones, ya que la evidencia actual no es suficiente para recomendar su uso en el tratamiento de la hipertensión arterial.

- Potasio: existe evidencia de que en la dieta de la mayoría de los individuos coexisten una baja ingesta de potasio, acompañando a una alta ingesta de cloruro de sodio. Existe consenso que dietas con contenidos más altos o aportes adicionales de cantidades moderadas de potasio, se asocian con PA más bajas, pudiendo inclusive disminuir la PA cuando está elevada ${ }^{80,81}$. Es así que el aporte de potasio podría aportar, salvo en algunas condiciones clínicas particulares, un efecto beneficioso sobre la presión arterial y mejoría del perfil metabólico. Sin embar- 
go, los contenidos de potasio de las aguas naturales suelen ser muy bajos, por lo que implicancia clínica de estos aportes es dudosa.

- Selenio:el exceso de selenio determinaría una elevación de 4,3 mm Hg de la sistólica y 1,6 $\mathrm{mm} \mathrm{Hg}$ de la diastólica, así como una mayor incidencia de dislipemia y diabetes ${ }^{82}$. También se ha descripto una relación en $\mathrm{U}$ con la incidencia de eventos cardiovasculares ${ }^{83}$. Al igual que en tema anterior, la relevancia clínica parece no ser importante.

\section{5. ¿Cuál es la implicancia de tomar agua y no otras bebidas sobre la presión arterial?}

- Dado tanto su valor biológico positivo (lo que hace) como el negativo (lo que no hace), como también la accesibilidad, disponibilidad y costo, el agua es el líquido recomendado para mantener una adecuada hidratación tanto a nivel poblacional como individual cuando lo comparamos con otras bebidas ${ }^{84}$. La osmolaridad plasmática tiende a mantenerse constante, dentro de un amplio margen de ingesta de agua,dado que el riñón se ocupa de regular el ahorro o eliminación adaptándolo a la ingesta. Esto posibilita un amplio margen de ingesta.

- Se recomienda que para las personas de actividad promedio que vivan en climas templados la ingesta de agua sea entre 2,5 a 3 litros por día.

- El $80 \%$ de la hidratación diaria proviene del agua que bebemos, a ella contribuyen todos los tipos de bebidas ${ }^{85,86}$. En cuanto a la hidratación, no se ha comprobado que ninguna fuente de agua sea mejor que otra.

- Los alimentos, por ser hiperosmolares, parecen favorecer el desarrollo de aterosclerosis probablemente por aumentar el calor central del cuerpo ${ }^{86}$. El aporte concomitante de agua con los alimentos reduce la osmolaridad de la comida contribuyendo de esta manera a retrasar el desarrollo de arteriosclerosis ${ }^{87,88}$.

- Existen evidencias contundentes del aumento de la presión arterial por el consumo abusivo de bebidas alcohólicas ${ }^{89}$. Múltiples estudios observacionales muestran una clara asociación entre ingesta de alcohol y aumento de la $\mathrm{PA}^{89,90}$. Los estudios de intervención sobre el efecto de la reducción de la ingesta de alcohol y reducción de PA aunque son de menor calidad, muestran resultados en el mismo sentido. Un meta-análisis de 15 estudios controlados randomizados ( N $=2234)$, en los que la reducción del consumo de alcohol fue la única intervención, mostró luego de 8 semanas promedio de seguimiento, una reducción significativa de 3.3 y $2.0 \mathrm{mmHg}$ en la PAS y PAD, respectivamente; el mismo estudio encontró una relación dosis-respuesta entre reducción de ingesta de alcohol y el descenso de $\mathrm{PA}^{91}$.

- El consumo habitual de agua en reemplazo de bebidas azucaradas (jugos, gaseosas y otras bebidas) puede reducir la cantidad de calorías ingeridas, y es adecuado y existe un meta-análisis que lo confirma ${ }^{92}$. Además, la ingestión de agua por sí misma puede reducir el riesgo de sobrepeso, especialmente cuando se la consume junto con los alimentos ${ }^{93,94}$.

- En relación al consumo de bebidas azucaradas, particularmente aquellas endulzadas con jarabe de maíz con alto contenido de fructosa no sólo aumentan la presión arterial y la incidencia de hipertensión arterial, sino también facilitan la instalación de todos los componentes del síndrome metabólico. Esto ha sido confirmado tanto en modelos animales como en el consumo humano, donde favorecen su instalación a las pocas semanas de uso ${ }^{95,96}$.

- Otras bebidas de consumo creciente en los últimos años vinculadas con el aumento de la presión arterial son las denominadas "bebidas energizantes". Estas bebidas, si bien tienen formulas variables, todas contienen esencialmente cantidades importantes de cafeína: entre 50 y $80 \mathrm{mg} / 100 \mathrm{~mL}$ (similar a una taza de café); y dado que se ingieren cuanto menos 4 o 5 veces esa cantidad, se sobrepasa rápidamente el límite superior de lo recomendado para el hombre adulto. Estas bebidas también contienen cantidades importante de azúcares, algunas de ellas tienen fructosa, y aminoácidos como taurina. La incorporación de cantidades elevadas de cafeína produce un aumento agudo de la presión arterial y riesgo de arritmias ${ }^{97}$. Por otro lado, las ingestas moderadas de café no parecen tener efectos crónicos perjudiciales sobre la presión arterial ni el riesgo cardiovascular.

- El agua es el reemplazo natural de las demás bebidas, que utilizadas en exceso, producen importantes perjuicios en la salud humana.

- Existen también evidencias de que beber una cantidad de agua superior a lo habitual puede tener un ligero efecto presor, que es mayor en pacientes con disfunción autonómica y con tendencia a la hipotensión, por lo menos en períodos $\operatorname{cortos}^{98}$. Si esto persiste en el tiempo es dudoso.

\section{6. ¿El sabor del agua según el contenido de mi- nerales influye sobre la adherencia a su con- sumo habitual?}

- El agua se define característicamente como un líquido incoloro, inodoro e insípido, pero en 
realidad no es así. El agua tiene características organolépticas específicas dependiendo de la presencia variable de componentes inorgánicos o sales minerales, de la existencia de componentes orgánicos, y también influyen los gases como el $\mathrm{CO}_{2}{ }^{99-101}$. Esto se conoce como el sabor de las aguas, y podemos definirlo como la impresión sensorial global que las mismas producen al ingerirlas. El sabor está constituido por la suma del gusto, el olor y la sensación que produce en la boca. La suma de estímulos sensoriales que el agua despierta son consecuencia de la composición química de la misma, junto a la sensación que el contacto con el agua y la temperatura produce ${ }^{35}$. Habitualmente hacemos referencia al gusto del agua porque lo consideramos erróneamente sinónimo de sabor, desconociendo la suma de sensaciones.

- Los cationes calcio, magnesio, sodio y potasio están presentes en la mayoría de las rocas y sedimentos, por lo tanto son componentes predominantes en la mayoría de los acuíferos, de donde se extraen las aguas naturales utilizadas para consumo humano ${ }^{35}$. De los cationes depende esencialmente el sabor del agua, mientras que los aniones, cuando superan ciertas concentraciones, pueden modificar la intensidad del sabor $^{100,101}$. Cuando las concentraciones de calcio, magnesio y/o sodio son elevadas generan un gusto amargo y salado del agua, y también, aunque en menor grado,el gusto ácido, metálico o astringente. Las concentraciones bajas de calcio, en el rango entre 20 y $40 \mathrm{mg} / \mathrm{L}$, confieren al agua sabor más dulzón, más agradable que cuando la concentración de calcio supera $100 \mathrm{mg} / \mathrm{L}^{102}$.

- Las aguas que contienen el anión cloruro junto a calcio, magnesio y sodio son percibidas como desagradables a concentraciones más bajas que las que contienen anión sulfato, que requieren concentraciones más altas para tener un sabor desagradable. La intensidad del sabor del sodio es más baja con bicarbonato y con sulfato, y tiene mayor intensidad con el anión cloruro ${ }^{103}$.

- Las sustancias disueltas en el agua, como hierro, magnesio, zinc y manganeso, aun en pequeñas cantidades, pueden modificar de manera importante el sabor del agua y producir un sabor metálico o astringente, generando rechazo en los consumidores ${ }^{35}$.

- Los compuestos orgánicos, también suelen impartir sabores característicos al agua. Muchas veces el sabor desagradable aparece a concentraciones inferiores al límite de toxicidad. Los fenoles y los correspondientes compuestos clorados generados en procesos de depuración con cloro imprimen al agua gustos muy característi- cos. Otras sustancias que confieren sabor desagradable al agua son surfactantes, alcoholes, ésteres, ácidos grasos y aldehídos ${ }^{104,105}$.

- Las bajas concentraciones de potasio mejoran su aceptación, mientras que altas concentraciones de sodio la disminuyen ${ }^{35}$.

- Las aguas con una mineralización muy baja (inferior a $100 \mathrm{mg} / \mathrm{L}$ de sólidos totales disueltos o residuos secos) tienen un característico gusto amargo y metálico, y tienden a ser menos aceptables para el consumidor, mientras que las aguas con mineralización media son aguas de buen sabor y las mejor aceptadas por el consumidor ${ }^{106}$.

- El pH es otro indicador de la calidad del agua: un agua a $\mathrm{pH}$ bajo tiene un gusto ácido, mientras que un $\mathrm{pH}$ alto le imprime un sabor jabonoso. El pH óptimo en cuanto al sabor es de 6 a 7. La carbonatación y la temperatura del agua también influye en el sabor.

- El mercado actual produce constantemente bebidas para diversos paladares que contribuyen el consumo global de líquidos en el día, generando la posibilidad de favorecer la ingesta de agua y minerales compitiendo con otras bebidas menos saludables. Quizás este sea unos de los motivos por los cuales el consumo de agua proveniente de fuentes naturales está creciendo en el mundo y también en nuestro país ${ }^{35,107}$.

- Teniendo en cuenta que el rechazo o aceptabilidad a la hora del consumo de aguas está impactado por su palatabilidad, éste es un punto esencial a la hora de promover su ingesta ${ }^{35,106-109}$.

\section{RESUMEN FINAL}

En este documento quisimos resaltar las siguientes consideraciones:

- El consumo habitualmente abusivo de cloruro de sodio tiene un rol central en el desarrollo y mantenimiento de la hipertensión arterial. Sin embargo la cantidad máxima de sodio aportada por aguas, ya sean de red o envasadas, es relativamente baja, menos del 10\% del consumo máximo recomendado por la Organización Mundial de la Salud. Es decir el aporte de sodio proveniente del agua, dentro de una alimentación variada, es porcentualmente poco relevante.

- Asimismo, la sal aportada por estas aguas no es cloruro de sodio sino otras sales como bicarbonato y sulfato de sodio, con un efecto diferente sobre la presión arterial.

- Cuando pensamos en cuáles son los elementos minerales ingeridos que influyen sobre la presión arterial y el riesgo cardiovascular, habitualmente pensamos sólo en el catión sodio como responsable, sin embargo este documen- 
to muestra evidencia que no es solamente el sodio. Está comprobado que el anión cloruro,acompañandoal sodio, es fundamental en el aumento de la presión arterial, cuando el anión acompañante no es el cloruro, los efectos del sodio sobre la presión arterial no parecen ser iguales. Existen estudios que muestran que cuando el anión acompañante del sodio es bicarbonato, se observa un efecto cuanto menos neutro, y quizás inverso, reduciendo la presión arterial.

- Los otros cationes, como el calcio, magnesio y potasio, que habitualmente están presentescuando las aguas provienen de fuentes naturales, deben ser considerados. Las cantidades de estos iones aportados con el agua es variable y depende de su origen. Estos cationes tendrían efectos contrarios sobre la presión arterial que los descriptos para el sodio, aunque esto requiere la adecuada confirmación clínica.

- No debemos olvidar que la concentración de minerales en las aguas se expresa en mg por litro y no en $\mathrm{mg}$ cada $200 \mathrm{ml}$, como en el resto de las bebidas y alimentos.

- Finalmente, el agua es el medio más adecuado para la hidratación del ser humano, indispensable para preservar la salud. El agua está libre de calorías, y con las ingestas habituales, el aporte de sodio no parece ser de una magnitud suficiente para provocar el desarrollo de enfermedad cardiovascular ni hipertensión arterial. Además, el consumo de agua puede prevenir la ingesta excesiva de bebidas alcohólicas o azucaradas, claramente más perjudiciales. El sabor agradable y composición adecuada del agua fa- cilita su consumo lo que contribuye a preservar y favorecer la salud humana.

\section{PARTICIPANTES DE LA TOMA DE POSICIÓN}

\section{Coordinación General: Dr. Felipe Inserra \\ Coordinador de contenidos de la pregunta 1 : \\ Dr. Martín Salazar}

Expertos: Dres. Raúl Echeverría, Hugo Farina, Irene

Ennis, Walter Espeche y Carlos March

Coordinador de contenidos de la pregunta 2:

Dr. Gustavo Lavenia

Expertos: Dres. Diego Nannini, Gustavo Blanco, Gustavo Staffieri, Mariana Radrizzani, Roberto Parodi, Jorgelina Presta

Coordinador de contenidos de la pregunta 3:

Dr. Alfredo Wassermann

Expertos: Dres. Lucas Aparicio, Paula Cuffaro, Walter Espeche, Diego Fernández, Gabriel Lapman

Coordinadores de contenidos de la pregunta 4:

Dres. Nicolás Renna, Roberto Miatello

Expertos: Dres. Claudio JooTuroni, María Peral de Bruno, Alejandro Saracco

Coordinador de contenidos de la pregunta 5:

Dr. Jorge Janson

Expertos: Dres. Gerardo Uceda, Carlos Galarza

Coordinador de contenidos de la pregunta 6:

Dr. Fernando Filippini

Expertos: Dres. Mario Groberman, Rosario Mariana Zucchi Araujo

Agradecemos la revisión final del manuscrito y las importantes sugerencias realizadas por el Dr. Daniel Piskorz

\section{BIBLIOGRAFÍA}

1. Intersalt Cooperative Research Group. Intersalt: an international study of electrolyte excretion and blood pressure. Results for 24 hour urinary sodium and potassium excretion. BMJ $1988 \mathrm{Jul}$ 30;297(6644):319-328.

2. Elliott P. Observational studies of salt and blood pressure. Hypertension 1991;17 [suppl 1]:13-18

3. Kaplan NM. Primary hypertension: pathogenesis. In: Kaplan NM, ed Kaplan's clinical hypertension. 9th ed. Philadelphia: Lippincott Williams \&Wilkins, 2006:50-121.

4. Denton D, Weisinger R, Mundy NI, et al. The effect of increased salt intake on blood pressure in chimpanzees. Nat Med 1995;1:1009-1016.

5. Ohigara T, Asano T, Fujita T. Contribution of salt intake to insulin resistance associated with hypertension. Life Sci 2003;73:509-523.

6. Van Vliet BN, Chafe LL, Halfyard SJ, Leonard AM. Distinct rapid and slow phases of salt-induced hypertension in Dahl salt-sensitive rats. J Hypertens 2006;24:1599-1606.

7. Van Vliet BN, Montani JP. The time course of salt-induced hypertension, and why it matters. Int J Obes (Lond) 2008; 32 Suppl 6:S35-S47. doi: 10.1038/ijo.2008.205.

8. Zhu J. Huang T. Lombard JH. Effect of high-salt diet on vascular relaxation and oxidative stress in mesenteric resistance arteries. J Vasc Res 2007; 44:382-390.

9. Hofman A, Hazebroek A, Valkenburg HA. A randomized trial of sodium intake and blood pressure in newborn infants. JAMA 1983;250:370-373.

10. He FJ, MacGregor GA. Importance of salt in determining blood pressure in children: meta-analysis of controlled trials. Hypertension 2006;48:861-869.

11. ElliottP,Stamler J, Nichols R, Dyer AR, Stamler R, Kesteloot H, et al. Intersalt revisited: further analyses of 24 hour sodium excretion and blood pressure within and across populations. BMJ 1996;312:1249-1255.

12. MacGregor GA, Markandu ND, Sagnella GA, Singer DR, Cappuccio FP. Double-blind study of three sodium intakes and long-term effects of sodium restriction in essential hypertension. Lancet 1989;334:1244-1247.

13. Sacks FM, Svetkey LP, VollmerWM, AppelLJ, Bray GA, HarshaD, et al. Effects on blood pressure of reduced dietary sodium and the Dietary Approaches to Stop Hypertension (DASH) diet. DASH-Sodium Collaborative Research Group. N Engl J Med 2001;344:3-10.

14. He FJ, MacGregor GA. Effect of modest salt reduction on blood pressure: a meta-analysis of randomized trials. Implications for public health. J Hum Hypertens 2002;16:761-770.

15. HeFJ, MacGregorGA. Effect oflonger-term modest saltreduction on blood pressure. Cochrane Database Syst Rev 2004;(3):CD004937.

16. He FJ, Li J, Macgregor GA. Effect of longer term modest salt reduction on blood pressure: Cochrane systematic review and meta-analysis of randomised trials. BMJ 2013 Apr 3;346:f1325.

17. Boudville N, Ward S, Benaroia M, House AA. Increased sodium intake correlates with greater use of antihypertensive agents by subjects with chronic kidney disease. Am J Hypertens 2005;18:1300 
18. Ram CV, Garrett BN, Kaplan NM. Moderate sodium restriction and various diuretics in the treatment of hypertension. Arch Intern Med 1981; 141:1015.

19. Singer DR, Markandu ND, Sugden AL, et al. Sodium restriction in hypertensive patients treated with a converting enzyme inhibitor and a thiazide. Hypertension 1991;17:798-803.

20. SlagmanMC, Waanders F, HemmelderMH, et al.Moderatedietarysodium restriction added to angiotensin converting enzyme inhibition compared with dual blockade in lowering proteinuria and blood pressure: randomised controlled trial. BMJ 2011;343:d4366.

21. Karppanen H. An antihypertensive salt: role of mildred seeling in its development. J Am Coll Nut 1994;13:493-495.

22. Tobian L, Jr., Binion JT. Tissue cations and water in arterial hypertension. Circulation 1952;5:754-758.

23. Wessels F, Losse H, Zumkley H. [Studies on the sodium content of erythrocytes in humans with normal and familial hypertensive blood pressure]. Verh Dtsch Ges Kreislaufforsch 1966;32:309-312.

24. Edmondson RP, Thomas RD, Hilton PJ, Patrick J, Jones NF. Abnormal leucocyte composition and sodium transport in essential hypertension. Lancet 1975;1:1003-1005.

25. Jaitovich A, Bertorello AM. Intracellular sodium sensing: SIK1 network, hormone action and high blood pressure. Biochimica et biophysica acta 1802:1140-1149, 2010.

26. De Lena SM, Echeverria RF, Escudero E, Gende OA, Cingolani HE. Blood pressure levels in young students. Correlation between body mass and metabolic and hemodynamic factors. Medicina 1996;56:161-168.

27. Hamlyn JM, Blaustein MP, Bova S, DuCharme DW, Harris DW, Mandel F, et al. Identification and characterization of a ouabain-like compound from human plasma. Proceedings of the National Academy of Sciences of the United States of America 1991;88:6259-6263.

28. BoedtkjerE, Aalkjaer C. IntracellularpHin the resistancevasculature: regulation and functional implications. J Vasc Res 2012:49:479-496.

29. Boedtkjer E, Praetorius J, Matchkov VV, Stankevicius E, Mogensen S, Füchtbauer AC, Simonsen U, Fuchtbauer EM, Aalkjaer C. Disruption of $\mathrm{Na}^{+}$, $\mathrm{HCO}^{-}$-cotransporter NBCn 1 (s/C4a7) inhibits NO-mediated vaso-relaxation, smooth muscle $\mathrm{Ca}^{2+}$-sensitivity and hypertension development in mice. Circulation 2011;124:1819-1829.

30. National Institute for Health and Clinical Excellence (NICE). Guidance on the prevention of cardiovascular disease at the population level. NICE, 2010. http://guidance.nice.org. uk/PH25.

31. The Task Force for the management of arterial hypertension of the European Society ofHypertension (ESH) and of the European Society of Cardiology (ESC)2013 ESH/ESC Guidelines for the management of arterial hypertension. J Hypertens 2013;31:1281-1357.

32. Institute of Medicine. Strategies to reduce sodium intake in the UnitedStates. April 2010. www.iom.edu/Reports/2010/Strategies-to-Reduce-Sodium-Intake-in-the-United-States.aspx

33. Schimieder RE, MesserliFH, Garavglia GE, Nuñez BS: Salt intake as a determinant of cardiac involvment in essential hypertension. Circulation 1988; 78:951-956

34. Tuomilehto J, Jousilahti P, Rastenyte D, Moltchanov V, Tanskanen A, Pietinen P, Nissinen A. Urinary sodium excretion and cardiovascular mortality in Finland: a prospective study. Lancet 2001:357:848-851.

35. Marcussen H, Holm PE, Hansen HChrB. Composition, flavor, chemical foodsafetyand consumer preferences of bottled water. Comprehensive Reviews in Food Science and Food Safety 2013;12:333-352.

36. Ministerio de Salud de la Argentina. http://www.prensa.argentina ar/2013/03/11/38976-salud-presento-informe-sobre-el-consumo-desal-en-el-pais.php

37. Catling LA, Abubakar I, Swift L, Hunter PR, Lake IR. A systematic review of analytical observational studies investigating the association between cardiovascular disease and drinking water hardness. I Water Health 2008;6:433-442.

38. EFSA Panel on Dietetic products, Nutrition, and Allergies (NDA). Scientific opinion on dietary reference values for water. European Food Safety Authority (EFSA), Parma, Italy. EFSA Journal 8:1459(48pp).2010.

39. Código Alimentario Argentino, Capítulo XII, Artículo 986-(Resolución Conjunta SPRel N²0/2010 y SAGyP N 106/2010).

40. Guidelines for drinking water quality; 4th edition; OMS; Ginebra, 2011. http://www.who.int/water_sanitation_health/publications/2011/ dwa chapters/en/
41. Schmidlin O, Tanaka M, Bollen AW, Yi SL, Morris RC Jr. Chloride-dominant salt sensitivity in the stroke-prone spontaneously hypertensive rat. Hypertension 2005;45:867-873.

42. Kotchen TA. Contributions of sodium and chloride to NaCl-induced hypertension. Hypertension 2005:45:849-850.

43. Schorr U, Distler A, Sharma AM. Effect of sodium chloride and sodium bicarbonaterich mineral water on blood pressure and metabolic parameters in elderly normotensive individuals: a randomized doubleblind crossover trial. J Hypertens 1996;14:131-135.

44. Tubek S. Role of trace elements in primary arterial hypertension: is mineral water style or prophylaxis? Biol Trace Elem Res 2006;114(1-3):1-5.

45. Santos A, Martins MJ, Guimarães JT, Severo M, Azevedo I. Sodium-rich carbonated natural mineral water ingestion and blood pressure. Rev Port Cardiol 2010;29:159-172.

46. Schoppen S, Pérez-Granados AM, CarbajalA, OubiñaP, Sánchez-MunizFJ, Gómez-Gerique JA, Vaquero MP. A sodium-rich carbonated mineral water reduces cardiovascular risk in postmenopausal women. J Nutr 2004; 134:1058-1063.

47. Zair Y, Kasbi-Chadli F, Housez B, Pichelin M, Cazaubiel M, Raoux F, Ouguerram K. Effect of a high bicarbonate mineral water on fasting and postprandial lipemia in moderately hypercholesterolemic subjects: a pilot study. Lipids Health Dis 2013;12:105-112.

48. Luft FC, Zemel MB, Sowers JA, Fineberg NS, Weinberger MH. Sodium bicarbonate and sodium chloride: Effects on blood pressure and electrolyte homeostasis in normal and hypertensive man. J Hypertens. 1990;8:663-670.

49. Mahajan A, Simoni J, Sheather SJ, Broglio KR, Rajab MH, Wesson DE. Daily oral sodium bicarbonate preserves glomerular filtration rate by slowing its decline in early hypertensive nephropathy. Kidney Int 2010;78:303-309.

50. Mandel El, Forman JP, Curhan, GC, Taylor EN. Plasma bicarbonate and odds of incident hypertension. Am J Hypertens 2013;26:1405-1412.

51. Bresson JL, Flynn A, Heinonen M, HulshofK, Korhonen H, Lagiou P, et al. EFSA-Q-2005-015a: Scientific Opinion of the Panel on Dietetic Products, Nutrition and Allergies on a request from the EC on dietary reference values for water. The EFSA Journal 2008;1-49.

52. Kousa A, Moltchanova E, Viik-Kajander M, Rytkönen M, Tuomilehto J, Tarvainen T, Karvonen M, for the Spat Study Group. Geochemistry of ground water and the incidence of acute myocardial infarction in Finland. J Epidemiol Community Health 2004;58:136-139.

53. Jee SH, Miller ER 3rd, Guallar E, Singh VK, Appel LJ, Klag MJ. The effect of magnesium supplementation on blood pressure: a meta-analysis of randomized clinical trials. Am J Hypertens 2002;15:691-696.

54. Arroyo JP, Gamba G. Advances in WNK Signaling of Salt and Potassium Metabolism: Clinical Implications. Am J Nephrol 2012;35:379-386.

55. Magnesium level in drinking water and cardiovascular risk factor: a hypothesis. J Am Coll Nutr 1994;13:493-495.

56. Beyer FR, Dickinson HO, Nicolson DJ, Ford GA, Mason J. Combined calcium, magnesium and potassium supplementation for the management of primary hypertension in adults. Cochrane Database Syst Rev 2006;19;(3):CD004640.

57. Suter PM, Sierro C, Vetter W. Nutritional factors in the control of blood pressure and hypertension. Nutr Clin Care 2002;5:9-19.

58. Dickinson HO, Nicolson DJ, Campbell F, Cook JV, Beyer FR, Ford GA, Mason J. Magnesium supplementation for the management of essential hypertension in adults. Cochrane Database Syst Rev. 2006;Jul 19;(3):CD004640.

59. Rylander $R$, Arnaud M. Mineral water intake reduces blood pressure among subjects with low urinary magnesium and calcium levels. BMC Public Health 2004;4:56.

60. Cunha AR, Umbelino B, Correia ML, Neves MF. Magnesium and vascular changes in hypertension. Int J Hypertens 2012;2012:754250. DOl: 10.1155/2012/754250

61. Monarca S, Donato F, Zerbini I, Calderon RL, Craun GF. Review of epidemiological studies on drinking water hardness and cardiovascular diseases. Eur J Cardiovasc Prev Rehabil 2006;13:495-506.

62. Yang CY. Calcium and magnesium in drinking water and risk of death from cerebrovascular disease. Stroke 1998;29:411-414.

63. Eisenberg MJ. Magnesium deficiency and sudden death. Am Heart J 1992;124:544-549.

64. Cunha AR, Medeiros F, Umbelino B, Oigman W, Touyz RM, Neves MF. Altered vascular structure and wave reflection in hypertensive women with low magnesium levels. J Am Soc Hypertens 2013;7:344-352. 
65. Rasic-Milutinovic Z, Perunicic-Pekovic G, Jovanovic D, Gluvic Z, Cankovic-Kadijevic M. Association of blood pressure and metabolic syndrome components with magnesium levels in drinking water in some Serbian municipalities. J Water Health 2012;10:161-169.

66. Marx A, Neutra RR. Magnesium in drinking water and ischemic heart disease. Epidemiol Rev 1997;19:258-272.

67. ChiuveSE, Sun Q, Curhan GC, Taylor EN, Spiegelman D, WillettWC, Manson $J$ J, Rexrode KM, Albert CM. Dietary and plasma magnesium and risk of COronary heartdisease among women. JAm Heart Assoc. 2013;2: e000114, doi: 10.1161/JAHA.113.000114

68. Whelton PK, Klag MJ. Magnesium and blood pressure: review of the epidemiologic and clinical trial experience. Am J Cardiol 1989;63:26G-30G.

69. Garzon P, Eisenberg MJ. Variation in the mineral content of commercially available bottled waters: implications for health and disease. Am J Med 1998; 105:125-130.

70. Catling LA, Abubakar I, Lake IR, Swift L, Hunter PR. A systematic review of analytical observational studies investigating the association between cardiovascular disease and drinking water hardness. J Water Health 2008, 6:433-442.

71. Morris RW, Walker M, Lennon LT, Shaper AG, Whincup PH. Hard drinking water does not protect against cardiovascular disease: new evidence from the British Regional Heart Study. Eur J Cardiovasc Prev Rehabil 2008;15:185-189.

72. Kass L, Weekes J, Carpenter L. Effect of magnesium supplementation on blood pressure: a meta-analysis. Eur J Clin Nutr 2012 Apr;66(4):411-418.

73. Larsson SC, Orsini N, WolkA. Dietary magnesium intake and risk of stroke: a meta-analysis of prospective studies. Am J Clin Nutr 2012;95:362-366.

74. Li K, Kaaks R, Linseisen J, Rohrmann S. Associations of dietary calcium intake and calcium supplementation with myocardial infarction and stroke risk and overall cardiovascular mortality in the Heidelberg cohort of the European Prospective Investigation into Cancer and Nutrition study (EPIC-Heidelberg). Heart 2012;98:920-925.

75. Allender PS, Cutler JA, Follmann D, Cappuccio FP, Pryer J, Elliott P. Dietary calcium and blood pressure: a meta-analysis of randomized clinical trials. Ann Intern Med 1996;124:825-831.

76. Goldberg GR, Jarjou LMA, Cole TJ, Prentice A. Randomized, placebo-controlled, calcium supplementation trial in pregnant Gambian women accustomed to a low calcium intake: effects on maternal blood pressure and infant growth. Am J Clin Nutr 2013;98:972-982.

77. van Mierlo LA, Arends LR, Streppel MT, Zeegers MP, Kok FJ, Grobbee DE, et al. Blood pressure response to calcium supplementation: a meta-analysis of randomized controlled trials.J Hum Hypertens 2006;20:571-580.

78. Larsson SC, Orsini N, Wolk A. Dietary calcium intake and risk of stroke: a dose-response meta-analysis. Am J Clin Nutr 2013;97:951-957.

79. Kh R, Khullar M, Kashyap M, Pandhi P, Uppal R. Effect of oral magnesium supplementation on blood pressure, platelet aggregation and calcium handling in deoxycorticosterone acetate induced hypertension in rats. $J$ Hypertens 2000;18:919-926.

80. Aburto NJ, Hanson S, Gutierrez H, Hooper L, Elliott P, Cappuccio FP. Effect of increased potassium intake on cardiovascular risk factors and disease: systematic review and meta-analyses. Br Med J. 2013 Apr 3;346:f1378

81. Adrogué HJ, Madias NE. Sodium and potassium in the pathogenesis of hypertension. N Engl J Med 2007;10;356:1966-1978.

82. Laclaustra M, Navas-Acien A, Stranges S, Ordovas JM, Guallar E. Serum selenium concentrations and hypertension in the US Population. Circ Cardiovasc Qual Outcomes 2009;2:369-376.

83. Flores-Mateo G, Navas-Acien A, Pastor-Barriuso R, GuallarE., Selenium and coronary heart disease: a meta-analysis. Am J Clin Nutr 2006;84:762-773.

84. Negoianu D, Goldfarb S. Justadd water. JAm Soc Nephrol 2008;19:1041-1043.

85. Institute of Medicine of the National Academies. Dietary Reference Intakes: Water, Potassium, Sodium, Chloride, and Sulfate.

86. Institute of Medicine (U.S.). Panel on Dietary Reference Intakes for Electrolytes and Water: DRI, Dietary Reference Intakes for Water, Potassium Sodium, Chloride, and Sulfate. Washington, DC, National Academies Press, 2004

87. Mathur RK. The role of hyperosmolar food in the development of atherosclerosis. Med Hypotheses 2005;64:579-581.

88. MathurRK. Role ofdiabetes, hypertension, and cigarettesmokingonatherosclerosis. J Cardiovasc Dis Res 2010;1:64-68.
89. Abramson JL, Lewis C, Murrah NV. Relationship of self-reported alcohol consumption to ambulatory blood pressure in a sample of healthy adults. Am J Hypertens 2010;23:994-999.

90. Ohira T, Tanigawa T, Tabata M, Imano H, Kitamura A, Kiyama M, Sato S, Okamura T, Cui R, Koike KA, Shimamoto T, Iso H. Effects of habitual alcohol intake on ambulatory blood pressure, heart rate, and its variability among Japanese men. Hypertension 2009:53:13-19.

91. Xin X, He J, Frontini MG, Ogden LG, Motsamai OI, Whelton PK. Effects of alcohol reduction on blood pressure: a meta-analysis of randomized controlled trials. Hypertension 2001;38:1112-1117.

92. Malik V, Popkin B, Bray G, Depres J-P, Willett W, Hu F. Sugar Sweetened Beverages and Cardiometabolic Risk: A Meta-analysis. Nutr Rev 2010;68: 439-458.

93. Stanhope KL, Havel PJ. Endocrine and metabolic effects of consuming beverages sweetened with fructose, glucose, sucrose, or high-fructose corn syrup. Am J Clin Nutr 2008;88:1733-1737.

94. Muckelbauer R, Libuda L, Clausen K, Toschke AM, Reinehr T, Kersting M. Promotion and provision of drinking water in schools for overweight prevention: randomized, controlled cluster trial. Pediatrics 2009; 123:661-667.

95. Hwang IS, Ho H, Hoffman BB. Fructose-induced insulin resistance and hypertension in rats. Hypertension 1987; 10:512-516.

96. Ferder L, Ferder MD, Inserra F. The role of high-fructose corn syrup in metabolic syndrome and hypertension. Curr Hypertens Rep 2010;12:105-112.

97. Mesas AE, Leon-Muñoz LM, Rodriguez-Artalejo F, Lopez-Garcia E. The effect of coffee on blood pressure and cardiovascular disease in hypertensive individuals: a systematic review and meta-analysis. Am J ClinNutr 2011:94:1113-1126.

98. Jormeus A, KarlssonSI, Dahlgren C, Lindström T, Nyström F. Doubling of Water Intake Increases Daytime Blood Pressure and Reduces Vertigo in Healthy Subjects. Clin Exp Hypertens 2010;32:7439-7443.

99. Rivera JA, Muñoz-Hernández O, Rosas-Peralta M, Aguilar-Salinas CA, Popkin BM, Willett WC. Beverage consumption for a healthy life: recommendations for the Mexican population. 2008.

100. Whelton AJ.Advancing Potable Water Infrastructure through an Improved Understanding of Polymer Pipe Oxidation, Polymer-Contaminant Interactions, and Consumer Perception of Taste. 2009.

101. Gilbertson TA, Baquero AF, Spray-Watson KJ. Water taste: the importance of osmotic sensing in the oral cavity. Journal of Water and Health. 04 suppl. 2006.

102. Lawless HT, Rapacki F, Horne J, Hayes A. The taste of calcium and magnesium salts and anionic modifications. Food Qual Pref 2003;14:319-325.

103. Koseki M, Fujiki S, Tanaka Y, Noguchi H, Nishikawa T. Effect of water hardness on the taste of alkaline electrolyzed water. J Food Sci 2005;70:5249-S253

104. Zoeteman BCJ. Drinking water taste and inorganic constituents. In: Zoeteman BCJ, editor. Sensory assessment of water quality. Oxford: Pergamon Press Ltd. p 55-65. 1980

105. Bruvold WH, Gaffey WR. Evaluative ratings of mineral taste inwater. Percept Motor Skill 1969:28:179-192.

106. Osmolalidad de Bebidas de Consumo Frecuente. Elizabeth Dini-G., Jorge De Abreu-C. y Emeris López-M. Mundo Alimentario. Centro de Atención Nutricional Infantil Antímano, CANIA, Caracas, Venezuela. Diciembre 2012.

107. World health organization: water sanitation health. Drinking-water quality. (On line). http://www.who.int/water_sanitation_health/dwq/ gdwa3_es_10.pdf

108. Teillet E, Urbano C, Cordelle S, Schlich P. Consumer perception and preference of bottled and tap water. J Sens Stud 25:463-480, 2010

109. Evaluación de las aguas comercializadas y consumidas en la Ciudad de Buenos Aires. Silvia Vilanova. Revista de la Maestría en Salud Pública ISSN 1667-3700 Año 2 - No 3. Agosto de 2004

110. Iglesias $R$, Carmuega E, Spena L, Casávola C. Creencias, mitos y realidades relacionadas al consumo de agua. Rev Insuficiencia Cardíaca, 2013, vol $8, N^{\circ} 2$.

111. Advancing PotableWaterInfrastructurethrough an Improved Understanding of Polymer Pipe Oxidation, Polymer-Contaminant Interactions, and Consumer Perception of Taste Whelton Andrew James. Dissertation submitted to the Faculty of the Virginia Polytechnic Institute and State University. April 2,2009. 